\title{
Kettler, David, und Thomas Wheatland (2019): Learning from Franz L. Neumann. Law, Theory, and the Brute Facts of Political Life
}

London: Anthem Press. 510 Seiten. \$299,95

\section{Hubertus Buchstein}

Angenommen: 23. September 2021 / Online publiziert: 12. Oktober 2021

(C) Der/die Autor(en) 2021

Auf welcher Konferenz in Europa oder den USA man David Kettler in den vergangenen 30 Jahren traf - immer kam man an irgendeinem Punkt des Gesprächs mit ihm auf sein großes Vorhaben zu sprechen, ein umfassendes Buch über seinen ehemaligen universitären Lehrer Franz L. Neumann zu schreiben. Zusammen mit Thomas Wheatland, der kürzlich ein wunderbares Buch über die Geschichte der Frankfurter Schule im Exil veröffentlich hat, konnte er sein langjähriges Vorhaben schließlich rechtzeitig zu seinem 90. Geburtstag zu einem erfolgreichen Ende führen.

Franz L. Neumann (1900-1954) war ein begnadeter Arbeitsrechtler während der Weimarer Republik. Nach seiner Flucht aus Nazideutschland gehörte er zu den politisch wachsten Köpfen am ebenfalls exilierten Institut für Sozialforschung von Max Horkheimer, wo er auch den „Behemoth“, seine monumentale Studie über Nazideutschland, schrieb. Er wechselte danach in den amerikanischen Behördendienst, um im Krieg gegen Deutschland und für den Neuaufbau einer demokratischen Ordnung aktiv mitzuwirken. Als Erster aus der Gruppe der „Frankfurter“ erhielt Neumann 1948 in den USA eine Professur. An der New Yorker Columbia Universität gehörte der 1930 in Leipzig geborene Kettler zu Neumanns Studierenden. Die ihn prägende Zeit mit Neumann währte allerdings nur wenige Semester. Denn im August 1954 verstarb Neumann bei einem Verkehrsunfall in der Schweiz.

Neben Otto Kirchheimer gilt Neumann heute als wichtigster Vertreter der Politikwissenschaft aus der ersten Generation der Kritischen Theorie der Frankfurter Schule. Zwar gibt es von ihm noch keine philologisch abgesicherte Edition seiner gesammelten Schriften, seine wichtigsten Beiträge sind aber bis heute sowohl in englischer wie deutscher Sprache leicht zugänglich geblieben und zum Teil mehrfach

Hubertus Buchstein $(\bowtie)$

Institut für Politik- und Kommunikationswissenschaft, Universität Greifswald, Greifswald,

Deutschland

E-Mail: buchstei@uni-greifswald.de 
neu aufgelegt. Auch an Sekundärliteratur zu Neumann - sei es in rekonstruktiver oder in aktualisierender Absicht - mangelt es auf beiden Seiten des Atlantiks nicht.

Das Buch ist im Aufbau konventionell und referiert auf zehn Kapitel verteilt in chronologischer Folge nahezu sämtliche Schriften Neumanns. Sehr ausführlich widmen sich die beiden Verfasser zunächst Neumanns rechtssoziologischer Promotionsschrift über das Verhältnis von Sein und Sollen. Auf knapp 40 Seiten kann man dann Zusammenfassungen seiner Beiträge zum Arbeitsrecht sowie zur Politik und Verfassung der Weimarer Republik nachlesen. Größeren Neuigkeitswert bietet dann aber das nahezu 100 Seiten umfassende Kapitel über Neumanns erstes Exil in London (1933-1936), in dem seine akademischen und politischen Kontexte an der London School of Economics genauer ausgeleuchtet werden.

Gut herausgestellt wird in den darauffolgenden drei Kapiteln Neumanns aktive Rolle am Horkheimer-Institut in New York, insbesondere als Initiator der Forschungsprojekte über den Antisemitismus, die das Institut später berühmt machen sollten. Das lange Kapitel über den „Behemoth“ besteht im Wesentlichen aus einem langatmigen Referat des Buchinhalts sowie einer Wiederholung der bekannten Kontroversen mit Horkheimer et al. über die Staatskapitalismusthese. Andere Fragen werden gar nicht erst gestellt, z. B.: Wer von den Mitarbeitern am Institut hatte bei welchen Kapiteln des Buches welche Anteile? Wie kam es eigentlich zu dem Titel des Buches? (Ossip K. Flechtheim, der auch an der Arbeit für das Buch beteiligt war, hat mir gegenüber einmal die Vermutung geäußert, Kirchheimer und Neumann hätten den Titel gemeinsam ausgeheckt, um Carl Schmitt damit zu ärgern.)

Demgegenüber bietet das neunte Kapitel über Neumanns Zeit beim Office of Strategic Services insofern Neues, als Kettler und Wheatland aufzeigen können, wie sich mit Neumanns Arbeitsaufnahme die Einschätzungen der amerikanischen Behörden über Nazideutschland änderten. In diesem Zusammenhang finden sich auch einige klug abwägende Überlegungen zu den erst in den 1990er-Jahren bekannt gewordenen indirekten Kontakten Neumanns mit dem KGB. Die dem Buch den Titel gebende Formulierung der ,,brute facts of political life“ bezieht sich auf die bittere Einsicht im Jahre 1944, dass es aus dem Inneren Deutschlands keine erfolgversprechende Widerstandsbewegung gegen die politische Führung des Landes mehr geben werde.

Neumanns Rolle beim Aufbau der Politikwissenschaft in Deutschland nach 1945 ist kaum zu überschätzen. Kettler und Wheatland zeigen dies überzeugend am Beispiel der Freien Universität Berlin - wobei ich mir erneut einen Blick in die einschlägigen Archive, in denen Neumanns Tätigkeiten dokumentiert sind, gewünscht hätte.

Nach seinem Wechsel an die Columbia University publizierte und lehrte Neumann im Bereich der Politischen Theorie und Ideengeschichte. Eine der berührendsten Passagen des Buches ist auf den Seiten 388-390 das „,brief memoir“ von Kettler über Neumann als Universitätslehrer. Etwas hörgeschädigt, mit starkem deutschem Akzent, bei den Vorlesungen zuweilen etwas langweilig, aber immer dann hellwach und brillant, wenn es zu Diskussionen kam, wusste Neumann seine Studierenden zu motivieren und zu inspirieren. Trotz seiner distanzierten Art konnte er zu vielen Studierenden eine persönliche Beziehung aufbauen und gleichzeitig vermitteln, dass er sich mit Haut und Haaren der theoretischen Beschäftigung mit Politik verschrie- 
ben hatte. Liest man die Erinnerung von Kettler, dann verwundert es nicht, dass Neumann mit Raul Hilberg oder Herbert Deane später berühmt gewordene Schüler hatte.

Die politiktheoretischen Spätschriften Neumanns nehmen im Vergleich zum Frühwerk und dem Londoner Exil deutlich weniger Seiten ein. Das ist insofern schade, als mehrere dieser Aufsätze heute zum klassischen Kanon der Politischen Theorie gehören und kontrovers rezipiert worden sind. Die Kontroversen drehen sich u.a. darüber, inwieweit Neumanns Spätwerk Ausdruck persönlicher Resignation ist oder aber als produktiver Impuls für eine Kritische Theorie der Politik gelesen werden kann. Die schlichte zusammenfassende Schlussthese des Buches lautet, dass Neumann als ,intellectual“ mit all seinen ,evasions, errors, and perplexities“ (S. 467) gelesen werden solle.

Angesichts der Fülle an Literatur zu Neumann sollte ein weiteres Buch über ihn einen gewissen Originalitätswert erfüllen können. Eine solche Originalität kann auf dreierlei Art und Weise erzeugt werden. Erstens, indem bislang nicht gesichtetes Archivmaterial zum Sprechen gebracht wird und damit ein neues Licht auf Leben und Werk von Neumann fällt. Oder zweitens, indem in kritischer Auseinandersetzung mit der reichhaltigen Sekundärliteratur neue Teil- oder Gesamtinterpretationen des bekannten Werks präsentiert werden. Oder schließlich drittens, indem man zu systematisch weiterführenden Aktualisierungen von Denkmotiven Neumanns gelangt. Streng genommen erfüllt das vorliegende Buch keines der drei genannten Originalitätskriterien.

Der Preis, den die Anthem Press für das gedruckte Buch verlangt, ist prohibitiv und trägt zur weiteren Ausplünderung von Bibliotheksetats durch raffgierige Verlage bei.

Funding Open Access funding enabled and organized by Projekt DEAL.

Open Access Dieser Artikel wird unter der Creative Commons Namensnennung 4.0 International Lizenz veröffentlicht, welche die Nutzung, Vervielfältigung, Bearbeitung, Verbreitung und Wiedergabe in jeglichem Medium und Format erlaubt, sofern Sie den/die ursprünglichen Autor(en) und die Quelle ordnungsgemäß nennen, einen Link zur Creative Commons Lizenz beifügen und angeben, ob Änderungen vorgenommen wurden.

Die in diesem Artikel enthaltenen Bilder und sonstiges Drittmaterial unterliegen ebenfalls der genannten Creative Commons Lizenz, sofern sich aus der Abbildungslegende nichts anderes ergibt. Sofern das betreffende Material nicht unter der genannten Creative Commons Lizenz steht und die betreffende Handlung nicht nach gesetzlichen Vorschriften erlaubt ist, ist für die oben aufgeführten Weiterverwendungen des Materials die Einwilligung des jeweiligen Rechteinhabers einzuholen.

Weitere Details zur Lizenz entnehmen Sie bitte der Lizenzinformation auf http://creativecommons.org/ licenses/by/4.0/deed.de. 